\title{
Review of: "Copper Measurement"
}

Professor Jon Heylings ${ }^{1}$

1 Keele University

We have been measuring copper by ICP-OES for many years. Although the mass spec method is better for some elements like iron, titanium and silicon we found that optical emission is fine for achieving low limits of quantitation in human skin in OECD 428 studies on copper based biocides. The reason we are interested now at DT L, Keele University is that low copper levels in skin (and respiratory tract) may be associated with age and ethnicity in COVID-19 infection.

Professor Jon Heylings 